\title{
Technical note: PaGELL v.1.5: A flexible parametric program for the Bayesian analysis of longevity data within the context of animal breeding
}

\section{J. Casellas*1 and L. C. Brito†}

*Grup de Recerca en Millora Genètica Molecular Veterinària, Departament de Ciència Animal i dels Aliments, Universitat Autònoma de Barcelona, 08193 Bellaterra, Barcelona, Spain

†Departamento de Zootecnia, Universidade Federal de Viçosa, 36570-900 Viçosa, Brazil

\section{ABSTRACT}

This technical note presents the program PaGELL v.1.5 (Parametric Genetic Evaluation of Lifespan in Livestock), a flexible software program to analyze (right-censored) longevity data in livestock populations, with a special emphasis on the genetic evaluation of the breeding stock. This software relies on a parametric generalization of the proportional hazard model; more specifically, the baseline hazard function follows a Weibull process and flexibility is gained by including an additional time-dependent effect with the number of change points defined by the user. The program can accommodate 3 different sources of variation (i.e., systematic, permanent environmental, and additive genetic effects) and both fixed and time-dependent patterns (only for systematic and permanent environmental effects). Analyses are performed within a Bayesian context by sampling from the joint posterior distribution of the model, and model fit can be easily determined by the calculation of the deviance information criterion. Although this software has already been used on field data sets, its performance has been double-checked on simulated data set, and results are presented in this technical note. PaGELL v.1.5 was written in Fortran 95 language and, after compiling with the GNU Fortran Compiler v.4.7 and later, it has been tested in Windows, Linux, and MacOS operating systems (both 32- and 64-bit platforms). This program is available at http://www.casellas.info/files/pageII.zip.

Key words: animal breeding, longevity, proportional hazard model, software, Weibull

Received July 25, 2016.

Accepted May 15, 2017.

${ }^{1}$ Corresponding author: joaquim.casellas@uab.cat

\section{Technical Note}

Survival analysis by proportional hazard models (Cox, 1972) must be viewed as an emerging statistical tool in the animal science framework with remarkable applications to several domestic species (Ducrocq et al., 1988a,b, 2000; Sánchez et al., 2006; Fernàndez de Sevilla et al., 2008) and economically relevant traits (Schneider et al., 2005; Zavadilová and Štípková, 2013; Casellas, 2016). The weight of functional characters such as survival or longevity in selection indexes has increased during recent decades (Serenius et al., 2006; Forabosco et al., 2009), given their close relation with culling costs (Stott, 1994; Horn et al., 2012) and society's concern for the well-being of domestic animals (Camm and Bowles, 2000). Nevertheless, the implementation of systematic genetic evaluations for longevity traits has become difficult due to the shortage of flexible and computationally efficient software programs accounting for animal models. To the best of our knowledge, available software is limited to the Survival Kit v6 (Ducrocq et al., 2010). That program relies on a 2-step approach involving both frequentist (systematic effects) and Bayesian inference (random effects), and focuses on nonparametric (Cox, 1972; Prentice and Gloeckler, 1978) and pure-Weibull models (Ducrocq et al., 1988a,b). Some limitations have been suggested for the Survival Kit when fitting animal models by Laplace approximation in small populations (i.e., several generations of related females; Mészáros et al., 2010). Although alternative parametric approaches were developed (Noura and Read, 1990; Tarrés et al., 2005; Casellas, 2007), we lack specific software within the context of animal breeding.

This technical note aims to present PaGELL v.1.5 (Parametric Genetic Evaluation of Lifespan in Livestock), a versatile software to account for additive genetic, permanent environmental, and systematic sources of variation from complete or right-censored longevity data. This software implements the piecewise Weibull proportional hazard model previously devel- 
oped and tested by Casellas (2007) within a Bayesian context. The source code was written in Fortran95 and is available at http://www.casellas.info/files/pageII .zip; PaGELL v.1.5 has been tested in Windows, Linux, and MacOS operating systems on both 32- and 64bit platforms after compiling with the GNU Fortran compiler v.4.7 and later (Free Software Foundation, Cambridge, MA).

The analytical model underlying program PaGELL v.1.5 accommodated 2 different elements of variation on longevity data; that is, the baseline hazard function $\left[h_{0}(t)\right]$ and the regression covariates $\left[\exp \left(\mathbf{w}_{i}^{\prime} \boldsymbol{\theta}\right)\right]$ as follows:

$$
h_{i}(t \mid \boldsymbol{\theta})=h_{0}(t) \exp \left(\mathbf{w}_{i}^{\prime} \boldsymbol{\theta}\right),
$$

where $\mathbf{w}_{i}$ is the incidence column vector for the $i$ th individual and $\boldsymbol{\theta}$ is the column vector of systematic, permanent environmental, and additive genetic effects. As noted by Cox (1972), $h_{0}(t)$ is the instantaneous failure rate at time $t$, this illustrating the behavior of the analyzed population when all regression covariates are set to zero. Following Casellas (2007), the baseline hazard function was implemented on the basis of a Weibull distribution with appropriate shape $(\rho)$ and scale $(\lambda)$ parameters, as well as an additional time-dependent covariate $\left(\xi_{k}\right)$ changing its effect at a given change points $\left(\tau_{k}\right)$ :

$$
\begin{gathered}
h_{0}(t)=\lambda \rho(\lambda t)^{\rho-1}, \\
\xi_{k} \tau_{k}<t<\tau_{k+1} \text { and } k=1, \ldots, m,
\end{gathered}
$$

where $\tau_{1}=0$ and $\tau_{m+1}$ was the largest observed lifetime. The number of change points must be defined by the user before the analysis, and the subsequent goodness of fit of the model is systematically evaluated by the deviance information criterion (Spiegelhalter et al., 2002). This parameterization partially mimics Prentice and Gloeckler (1978) grouped data model as implemented in the Survival Kit (Mészáros et al., 2013); differences rely on (1) the use of a Weibull instead of an exponential regression model, and (2) the inclusion of the additional time-dependent effect changing at specific change-points instead of at the beginning of each new time interval (i.e., minor parameterization). Note that PaGELL v.1.5 can also analyze stratified data sets by assuming different $\rho, \lambda$, and change-points-related parameters for each stratum. On the other hand, PaGELL v.1.5 allows for a highly flexible definition of regression covariates including fixed and time-dependent systematic effects (both discrete and continuous), permanent environmental effects (fixed and time-dependent), and the additive genetic effect inherent to standard animal models for genetic evaluation. Only animal models can be accommodated, whereas other programs such as the Survival Kit must be considered if sire-related models are necessary (Ducrocq et al., 2010; Mészáros et al., 2013). There are no restrictions on the number of systematic and permanent environmental effects and the program can accommodate right-censored data as usual in standard survival analysis.

The analytical model is solved through Bayesian inference; apart from the Weibull-related a priori distribution of survival data and the expected Gaussian distributions assumed for permanent environmental and additive genetic effects, all remaining a priori distributions were assumed flat, as previously assumed by Damgaard and Korsgaard (2006a,b) and Sánchez et al. (2006). All the unknown parameters in the model are easily sampled from their joint posterior distribution by Monte Carlo Markov chains (MCMC), where the length of burn-in and sampling periods are defined by the user in the parameter file. The program requires 4 (space-delimited) input files: (1) parameter file (input/ output options and analysis settings), (2) pedigree file (3-column file identifying each individuals and its parents; all parents appear earlier than their offspring), (3) survival data file (survival data and incidence of the time-independent effects), and (4) time-dependent effects (optional; incidence of the time-dependent effects). Output files provide posterior means and standard deviations of all parameters of the model, both those involved in the baseline function and the regression covariates. All samples from the MCMC process are provided for variance components and baseline function parameters, to create posterior density figures or calculate alternative reference statistics such as credibility intervals and modal estimates, among others. These outputs can be easily transferred to MCMC diagnosis programs such as CODA (Plummer et al., 2006) to corroborate convergence of the sampling process.

The Bayesian parameterization implemented in PaGELL v.1.5 was fully described by Casellas (2007) based on Nguyen et al. (1984) and Noura and Read (1990), and model fit was tested on several simulation scenarios (Casellas, 2007). To put a special emphasis on genetic evaluation procedures, 2 different sets of simulated populations were generated. The first set involved a total of 1,000 populations, each involving 5 nonoverlapped generations with 400 sires and 1,600 dams mated at random. Each individual had a longevity record sampled from a piecewise Weibull distribution (Nguyen et al., 1984) with shape $(0.5<\rho<1.5)$ and scale $(0.0001<\lambda<0.001)$ parameters sampled at random between appropriate boundaries, and between 0 and 3 additional change points $\left(\tau_{k}\right)$ distributed at ran- 
dom along the parametric space; moreover, 2 systematic effects with 10 levels each (the magnitude of each level was sampled from a uniform distribution between -1 and 1) and the infinitesimal additive genetic effect $\left(\sigma_{a}^{2}\right.$; this was sampled at random between 0.1 and 1.1$)$ were included in the simulation process; note that the heritability on the original scale (Yazdi et al., 2002) fluctuated between 9 and $52 \%$. Finally, random censoring was also considered (0 to 50\%). The second set of simulated populations assumed the same structure and simulation parameters, only modifying the size of the population; this fluctuated between 500 and 5,000 individuals. Each data set was analyzed with PaGELL v.1.5 and assuming the true simulation model for systematic and additive genetic effects, as well as the number of change-points; a unique MCMC was launched with 500,000 iterations and after discarding the first 50,000 as burn-in. All analyses were performed with a server with 2 Intel Xeon E5-2400 processors with a CentOS 6.6 Linux operating system. In terms of central processing unit (CPU) time, the analysis of a 5,000 individualpopulation took slightly less than $35 \mathrm{~min}$. Although the CPU time demand for PaGELL v.1.5 depends on the length of MCMC, this is clearly higher than the CPU time reported for the different parameterizations implemented in the Survival Kit (e.g., Ducrocq et al., 2000).
This may discourage the use of PaGELL v.1.5 on large data sets, such as those involved in current genetic evaluation of dairy cattle for longevity-related traits (Vukasinovic et al., 2001; Caraviello et al., 2004).

As shown in Figure 1, the correlation coefficient between simulated and predicted $\sigma_{a}^{2}$ was high (0.947) and the linear regression fitted a unity line ( showing a reliable statistical behavior of program PaGELL v.1.5 when performing genetic evaluations for lifespan traits. Similar correlation coefficients $(>0.9)$ were obtained when comparing simulated and predicted $\rho$ and $\lambda$ parameters (results not shown). Moreover, Figure 2 shows the moderate effect of censoring percentage on the accuracy of predicted breeding values, suggesting a slight decrease when censoring increased. There were no evident trends for the accuracy of predicted breeding values based on $\rho$ and $\lambda$ parameters (results not shown). The correlation between simulated and predicted breeding values was moderate to high across a wide range of small populations (500 to 5,000 individuals; Figure 3); this pattern must be of special relevance for animal breeding purposes in small populations where other software may underperform.

This program can fit a wide range of survival-related patterns as reported by Casellas and Bach (2012), with this flexibility highlighting PaGELL v.1.5 as an

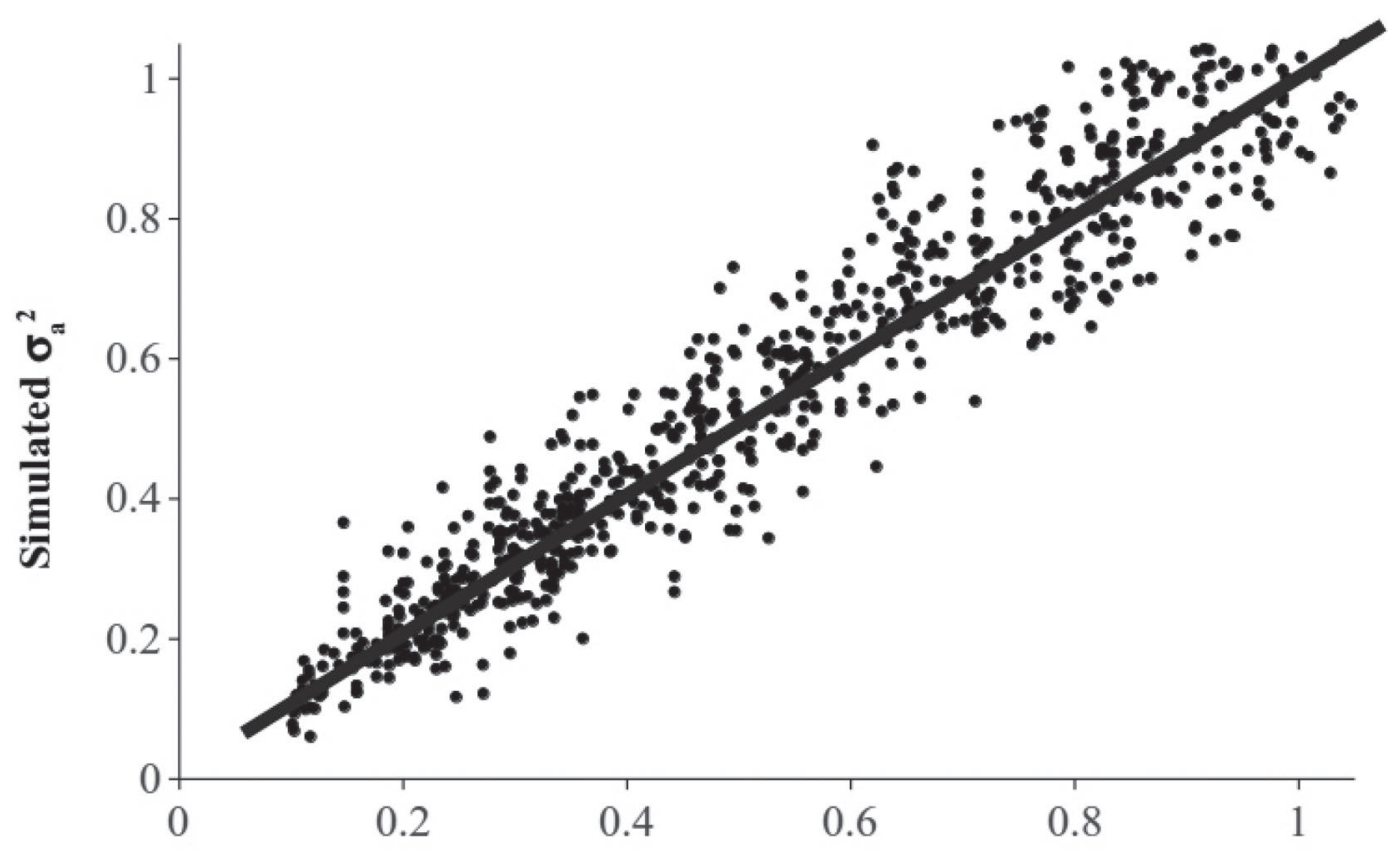

Predicted $\sigma_{\mathrm{a}}^{2}$

Figure 1. Scatterplot of simulated and predicted additive genetic variance $\left(\sigma_{a}^{2}\right)$ for longevity with program PaGELL v.1.5 in 5-generation populations with 400 sires and 1,600 dams per generation; a line with slope 1 has been drawn through the data points. 


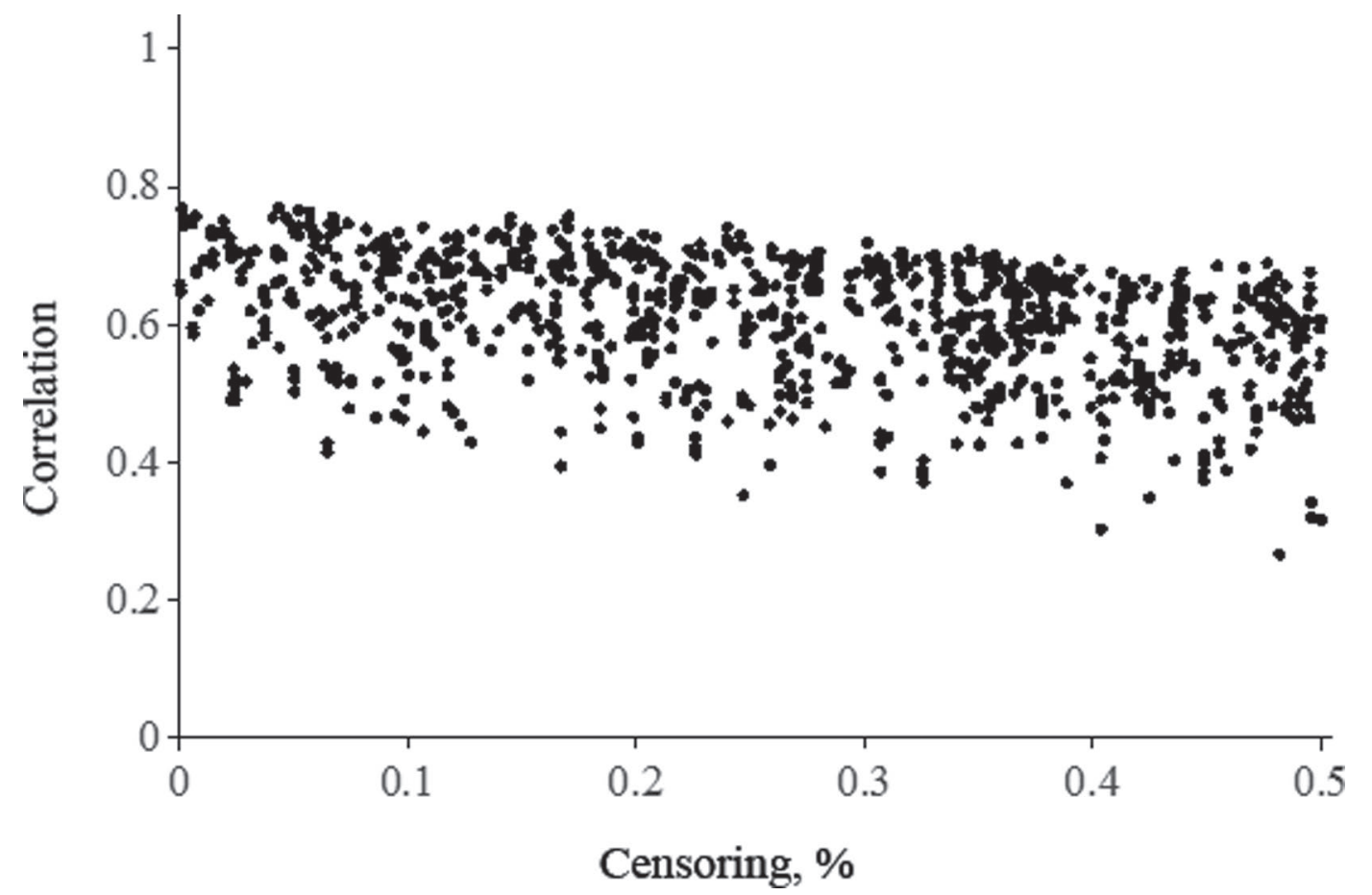

Figure 2. Correlation coefficient between simulated and predicted breeding values for longevity by the program PaGELL v.1.5, depending on the percentage of censoring applied on longevity data. A total of 1,000 populations were simulated, each involving 5 generations, with 400 sires and 1,600 dams per generation.

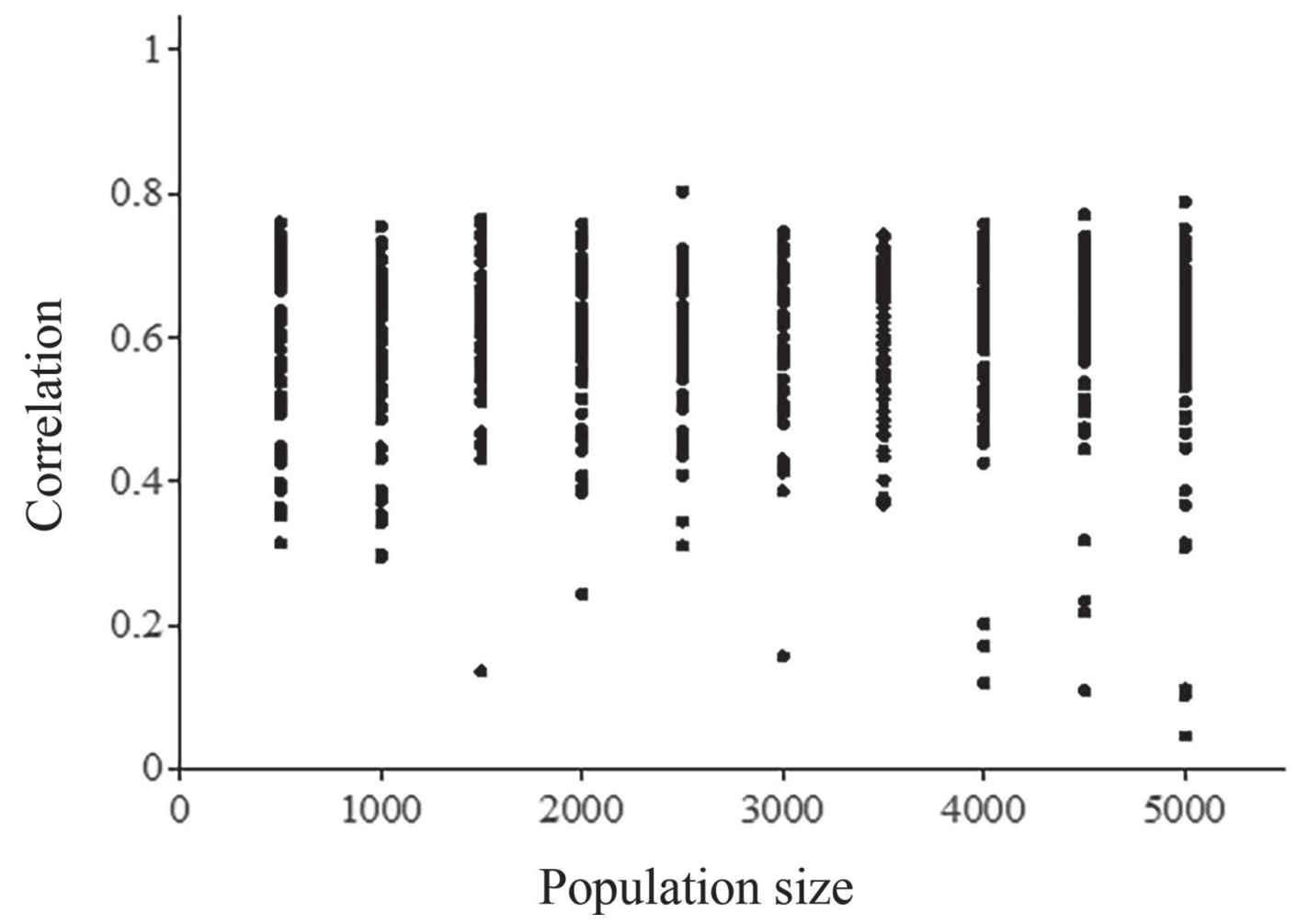

Figure 3. Correlation coefficient between simulated and predicted breeding values for longevity by the program PaGELL v.1.5, depending on the size of the data set (number of individuals). A total of 1,000 populations were simulated, each involving 5 generations, with a censoring percentage between 0 and $50 \%$. 
appealing statistical tool for survival analysis and its implementation for genetic evaluation of current breeding stock.

\section{ACKNOWLEDGMENTS}

The research contract of J. Casellas was partially financed by Spain's Ministerio de Ciencia e Innovación program Ramón y Cajal (Madrid, Spain; reference RYC-2009-04049).

\section{REFERENCES}

Camm, T., and D. Bowles. 2000. Animal welfare and the treaty of Rome-Legal analysis of the protocol on animal welfare and welfare standards in the European Union. J. Environ. Law 12:197-205.

Caraviello, D. Z., K. A. Weigel, and D. Gianola. 2004. Prediction of longevity breeding values for US Holstein sires using survival analysis methodology. J. Dairy Sci. 87:3518-3525.

Casellas, J. 2007. Bayesian inference in a piecewise Weibull proportional hazards model with unknown change points. J. Anim. Breed. Genet. 124:176-184.

Casellas, J. 2016. Comparison between linear and proportional hazard models for the analysis of age at first lambing in the Ripollesa breed. Animal 10:365-371.

Casellas, J., and R. Bach. 2012. Comparison of linear, skewed-linear, and proportional hazard models for the analysis of lambing interval in Ripollesa ewes. J. Anim. Sci. 90:1788-1797.

Cox, D. R. 1972. Regression models and life tables (with discussion). J. R. Stat. Soc. B 34:187-220.

Damgaard, L. H., and I. R. Korsgaard. 2006a. A bivariate quantitative genetic model for a linear Gaussian and a survival trait. Genet. Sel. Evol. 38:45-64.

Damgaard, L. H., and I. R. Korsgaard. 2006b. A bivariate quantitative genetic model for a threshold trait and a survival trait. Genet. Sel. Evol. 38:565-581.

Ducrocq, V., B. Besbes, and M. Protais. 2000. Genetic improvement of laying hens viability using survival analysis. Genet. Sel. Evol. $32: 23-40$.

Ducrocq, V., R. L. Quaas, E. Pollak, and G. Casella. 1988b. Length of productive life of diary cows. II. Variance component estimation and sire evaluation. J. Dairy Sci. 71:3071-3079.

Ducrocq, V., R. L. Quaas, E. Pollark, and G. Casella. 1988a. Length of productive life of dairy cows. I. Justification of a Weibull model. J. Dairy Sci. 71:3061-3070.

Ducrocq, V., J. Sölkner, and G. Mészáros. 2010. Survival Kit v6-A software package for survival analysis. Proc. 9th World Congr. Genet. Appl. Livest. Prod., Leipzig, Germany.

Fernàndez de Sevilla, X., E. Fàbrega, J. Tibau, and J. Casellas. 2008. Effect of leg conformation on survivability of Duroc, Landrace, and Large White sows. J. Anim. Sci. 86:2392-2400.
Forabosco, F., J. H. Jakobsen, and W. F. Fikse. 2009. International genetic evaluation for direct longevity in dairy bulls. J. Dairy Sci. 92:2338-2347.

Horn, M., W. Knaus, L. Kirner, and A. Steinwidder. 2012. Economic evaluation of longevity in organic dairy cows. Organic Agric. $2: 127-143$.

Mészáros, G., J. Pálos, V. Ducrocq, and J. Sölkner. 2010. Heritability of longevity in Large White and Landrace sows using continuous time and grouped data models. Genet. Sel. Evol. 42:13.

Mészáros, G., J. Sölkner, and V. Ducrocq. 2013. The Survival Kit: Software to analyze survival data including correlated random effects. Comput. Methods Programs Biomed. 110:503-510.

Nguyen, H. T., G. S. Rogers, and E. A. Walker. 1984. Estimation in change-point hazard rate models. Biometrika 71:299-304.

Noura, A. A., and K. L. Q. Read. 1990. Proportional hazards changepoint models in survival analysis. Appl. Stat. 39:241-253.

Plummer, M., N. Best, K. Cowles, and K. Vines. 2006. CODA: Convergence diagnosis and output analysis for MCMC. R News 6:7-11.

Prentice, R. L., and L. Gloeckler. 1978. Regression analysis of grouped survival data with application to breast cancer data. Biometrics 34:57-67.

Sánchez, J. P., I. R. Korsgaard, L. H. Damgaard, and M. Baselga. 2006. Analysis of rabbit doe longevity using a semiparametric lognormal animal frailty model with tie-dependent covariates. Genet. Sel. Evol. 38:281-295.

Schneider, M. P., E. Strandberg, V. Ducrocq, and A. Roth. 2005. Survival analysis applied to genetic evaluation for female fertility in dairy cattle. J. Dairy Sci. 88:2253-2259.

Serenius, T., K. J. Stalder, T. J. Baas, J. W. Mabry, R. N. Goodwin, R. K. Johnson, O. W. Robinson, M. Tokach, and R. K. Miller. 2006. National Pork Producers Council Maternal Line National Genetic Evaluation Program: a comparison of sow longevity and trait associations with sow longevity. J. Anim. Sci. 84:2590-2595.

Spiegelhalter, D. J., N. G. Best, B. P. Carlin, and A. van der Linde. 2002. Bayesian measures of model complexity and fit. J. R. Stat. Soc. B 64:583-639.

Stott, A. W. 1994. The economic advantage of longevity in the dairy cow. J. Agric. Econ. 45:113-122.

Tarrés, J., J. Casellas, and J. Piedrafita. 2005. Genetic and environmental factors influencing mortality up to weaning of Bruna dels Pirineus beef calves in mountain areas. A survival analysis. J. Anim. Sci. 83:543-551.

Vukasinovic, N., J. Moll, and L. Casanova. 2001. Implementation of a routine genetic evaluation for longevity based on survival analysis techniques in dairy cattle populations in Switzerland. J. Dairy Sci. 84:2073-2080.

Yazdi, M. H., P. M. Visscher, V. Ducrocq, and R. Thompson. 2002. Heritability, reliability of genetic evaluations and responses to selection in proportional hazards models. J. Dairy Sci. 85:1563-1577.

Zavadilová, L., and M. Štípková. 2013. Effect of age at first calving on longevity and fertility traits for Holstein cattle. Czech J. Anim. Sci. 58:47-57. 